\title{
A NEW HELICONIA (MUSACEAE) FROM SURINAME
}

\author{
P. J. M. MAAS and M. J. M. DE ROOIJ
}

Instituut voor Systematische Plantkunde, Utrecht

In the course of next year a revision of the surinam Musaceae will be published. A new Heliconia species recognized during this study, is described here.

Heliconia nickeriensis Maas \& de Rooij spec. nov. Fig. 1 and 2. Herba gracilis, 1-3(-4) $\mathrm{m}$ alta. Petiolus $22-60 \mathrm{~cm}$ longus. Lamina anguste ovata, 40-82 cm longa, 9-16 cm lata, basi (oblique) acuta, apice acuta ad leviter acuminata. Inflorescentia erecta, $10-20 \mathrm{~cm}$ longa, pedunculo viride, $35-40 \mathrm{~cm}$ longa, glabro. Rhachis leviter ad valde flexuosa, $0.2-0.3 \mathrm{~cm}$ crassa, glabra. Bracteae 3-7, rubrae, intus luteae, aurantiacae vel aurantiaco-rubrae, apice luteae, distichae, obtusae, anguste cymbiformes, glabrae. Bracteae basales $8.5-14 \mathrm{~cm}$ longae, (1.3-) $1.5-2.3 \mathrm{~cm}$ latae, secundae $6.5-10 \mathrm{~cm}$ longae, $1.3-2.2$ $\mathrm{cm}$ latae, . tiae $5-8.5 \mathrm{~cm}$ longae, $0.8-1.5 \mathrm{~cm}$ latae. Internodium basale $3-5 \mathrm{~cm}$ longum, secundum $2.7-3.5 \mathrm{~cm}$ longum. Bracteolae luteae, hyalinae, anguste triangulares, $4-4.2 \mathrm{~cm}$ longae, glabrae. Flores aurantiaci ad luteo-aurantiaci, leviter curvati, $4-5.5 \mathrm{~cm}$ longi, glabri, sepalo libero valde recurvato. Staminodium anguste obovatum, apiculatum, $0.8 \mathrm{~cm}$ longum, $0.3 \mathrm{~cm}$ latum. Ovarium luteum. Pedicelli luteo-albi, ad $1 \mathrm{~cm}$ longi; in stato fructifero ad $2 \mathrm{~cm}$ longi. Fructus ca $1 \mathrm{~cm}$ longus.

TYPE. P. J. M. \& H. Maas \& P. Teunissen 2366 (holotype, U; isotypes, BBS, CAY, F, GB, K, MO, NY, P, RB, VEN, Z), along ditch, $15 \mathrm{~km} \mathrm{~N}$ of Wageningen, distr. Nickerie, Suriname.

Distribution. Guyana and Suriname; all collected on clayey soils along ditches.

GuYana. Mulson Creek, Berbice, Grewal 268, 271 (U, UG); Edenburg, Berbice, Grewal 274(U,UG); Ithaca, Berbice, Grewal 279(U, UG); Richmond, 3.5 miles Line 28, W Coast, Essequibo, Harris, TP 152 (K); Anna Regina, $2^{1} / 4$ miles, W Coast, Essequibo, Harris, TP $515(\mathrm{~K})$.

SURINAME. Nanni Creek, distr. Nickerie, Hekking 965 (BBS, K, NY, U); 15 $\mathrm{km} \mathrm{N}$ of Wageningen, distr. Nickerie, P. J. M. \& H. Maas \& P. Teunissen 2366 (see type); 'Lekbeteugelingsdam', distr. Nickerie, Teunissen- LBB 14484 (BBS).

The closest relative is $H$. acuminata, but $H$. nickeriensis can be readily recognized by its obtuse, wider, orange (instead of red) bracts and by its orange flowers. 

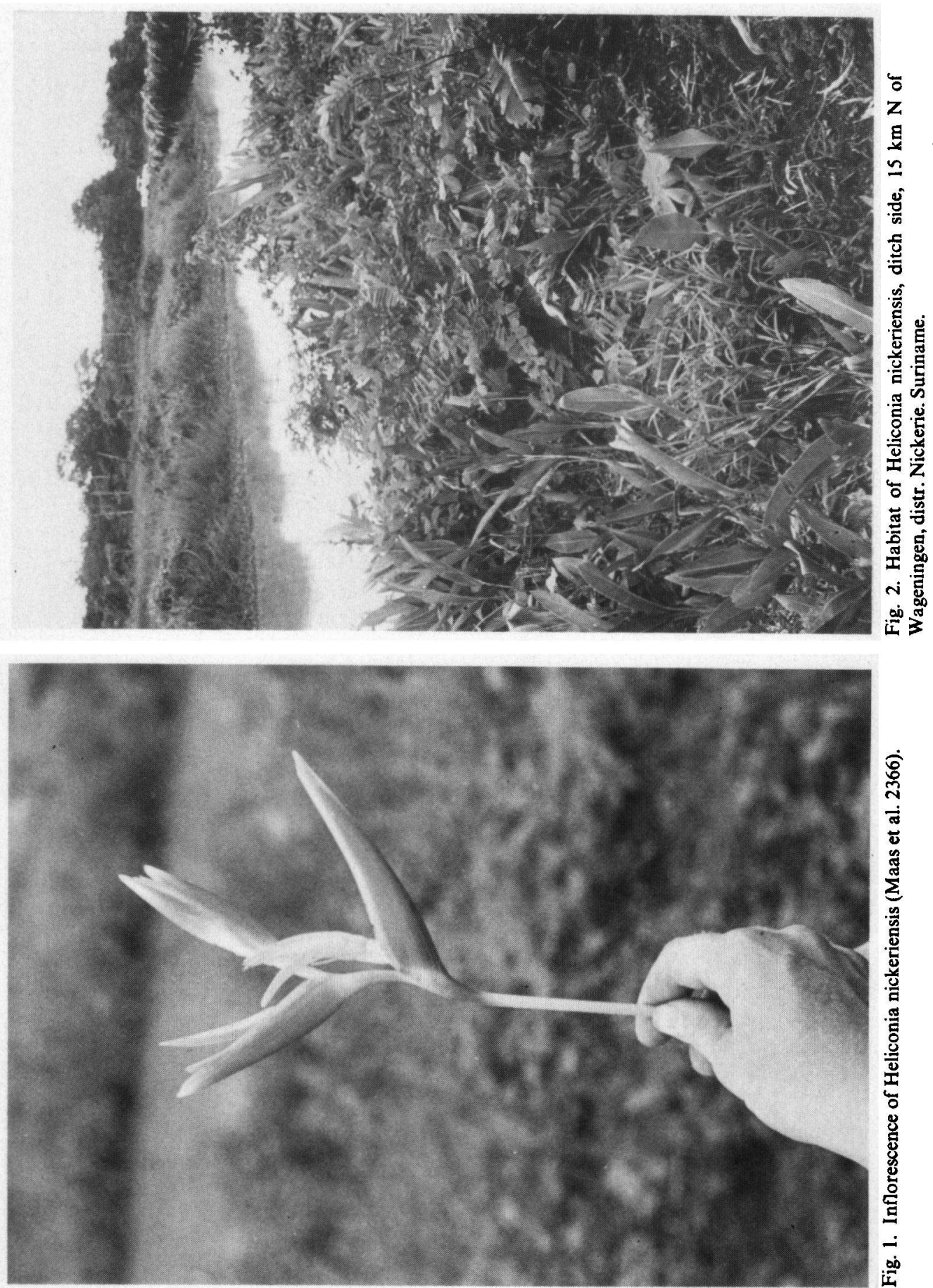